\title{
Modelling of spheroidal drop evaporation with non-uniform temperature conditions
}

\section{Gianpietro Elvio Cossali, Stefano Ravasio and Simona Tonini*}

\author{
Department of Engineering and Applied Sciences, University of Bergamo, Italy \\ *Corresponding author: simona.tonini@unibg.it
}

\begin{abstract}
The heating and evaporation of single component spherical and spheroidal drops in gaseous quiescent environment are predicted, accounting for the effect of a non-uniform distribution of the temperature at the drop surface. The analytical solution of the species conservation equations in the proper coordinate system (spherical/spheroidal) is implemented to numerically solve the energy equation in a rectangular domain. The effect of temperature non-uniformity on the local Nusselt number and global heat and evaporation rates is calculated for different species, drop deformation and gaseous temperature.
\end{abstract}

\section{Keywords}

Drop evaporation, spheroidal coordinates, non-uniform Dirichlet Boundary conditions.

\section{Introduction}

Most of the models predicting the drop heating and evaporation to be implemented in CFD codes for dispersed phase applications rely on the assumption that drops are spherical, thus allowing a simpler solution in spherical coordinates of the energy and species conservation equations. However, experimental investigation on liquid drops in multi-particle systems has revealed that they are subject to significant shape deformations while interacting with the carrier phase [1-3], due to the interaction of surface tension and fluid-dynamic stresses on the drop surface [3]. Numerical investigations on oscillating drops [4,5] have shown that the vapour and heat fluxes on the drop surface are not uniform and they were empirically correlated to the local mean curvature of the surface $[1,6]$. Analytical modelling of the heating and evaporation of spheroidal drops have shown that the local vapour and heat flux scale with the fourth root of the Gaussian curvature $[7,8]$ and later the same result was extended to a wider class of drop shapes [9].

When dynamical simulation of droplet heating and evaporation is necessary, uniform drop temperature is often assumed, on the basis of a commonly accepted belief that the internal recirculation would maintain uniform conditions. However a more accurate simulation can be obtained by using the concept of effective conductivity, firstly introduced by [10], to account for the effect of recirculation (see also [11] and [12]) and, although this cannot properly describe the temperature field inside the droplet, it can give a better estimation of the droplet surface temperature [13] .

Recent modelling of heating and evaporation of spheroidal droplets [14] revealed that the uneven distribution of fluxes on the drop surface causes a corresponding uneven distribution of temperature on the drop surface, during most of the drop lifetime. This non-uniform temperature distribution affects the heat and vapour flow fields in a non neglectful way.

The motivation of the work reported here is to investigate, through a combined analytical-numerical solution of the species and energy conservation equations, the effect of non-uniform Dirichlet boundary conditions at the drop surface (for spheroidal liquid drops) on the local heat and mass transfer coefficients.

\section{Mathematical modelling}

The evaporation of a single-component drop under quasi-steady conditions in a quiescent atmosphere, where the Stefan flow characterises the flow field, is described by the species conservation equations coupled with the energy conservation equation:

$\nabla_{j} n_{j}^{(p)}=0 \quad p=0,1$

where:

$n_{j}^{(p)}=\rho U_{j} \chi^{(p)}-\rho D_{10} \nabla_{j} \chi^{(p)} \quad p=0,1$ 
are the mass fluxes, $p=1$ stands for the evaporating component and $p=0$ for the gas. After summation, equations (1) yield the mass conservation equation:

$\nabla_{j} \rho U_{j}=0$

These three equations are then not independent and one of the species conservation can be disregarded. Moreover, assuming that the diffusion of component $p=0$ into the drop can be neglected, the mass flux of the component $p=0$ is nil at drop surface and then is nil everywhere. This last observation, after introducing the new variable: $G=\ln \left(1-\chi^{(1)}\right)$, and assuming constant properties, allows to write the conservation equations under the form:

$\nabla^{2} G=0$

(see also [9] for further details). The evaporation model is coupled with the energy equation, which has the form:

$L e^{-1} \nabla_{j} G \nabla_{j} T-\nabla^{2} T=0$

where $L e=\frac{k}{\rho D_{10} c_{p, v}}$ (see again [9] for further details).

\section{Spherical and spheroidal drops}

Equation (4) and (5) can be used to model heating and evaporation of drops of any shape, since the drop shape enters the problem through the boundary conditions. The case of spherical and spheroidal drops can be more easily treated solving the problem in proper coordinate systems. Using the spherical coordinates:

$x=R_{0} \frac{\sqrt{1-\eta^{2}}}{\zeta} \cos \varphi ; y=R_{0} \frac{\sqrt{1-\eta^{2}}}{\zeta} \sin \varphi ; z=R_{0} \frac{\eta}{\zeta}$

where:

$\zeta=\frac{R_{0}}{r} ; \eta=\cos \theta$

the drop surface of a spherical drop is defined by the equation $\zeta=\zeta_{0}$, and similarly in spheroidal prolate and oblate coordinates:

$$
\begin{aligned}
& x=a \sqrt{\zeta^{2}-1} \sqrt{1-\eta^{2}} \cos \varphi ; y=a \sqrt{\zeta^{2}-1} \sqrt{1-\eta^{2}} \sin \varphi ; z=a \zeta \eta \text { (prolate) } \\
& x=a \sqrt{\zeta^{2}+1} \sqrt{1-\eta^{2}} \cos \varphi ; y=a \sqrt{\zeta^{2}+1} \sqrt{1-\eta^{2}} \sin \varphi ; z=a \zeta \eta \quad \text { (oblate) }
\end{aligned}
$$

the drop surface (a prolate or oblate spheroid, see figure 1 ) is again defined by the equation $\zeta=\zeta_{0}$, but it must be noticed that now the coordinates $\zeta$ and $\eta$ have different definitions.

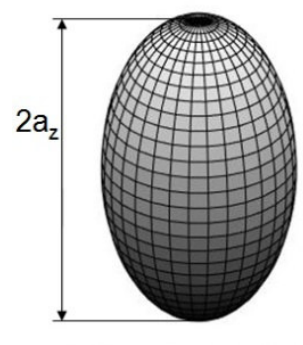

Prolate spheroid

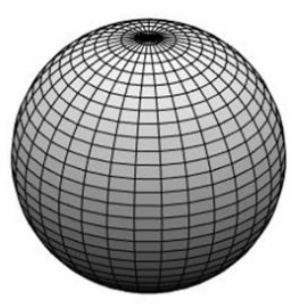

Sphere

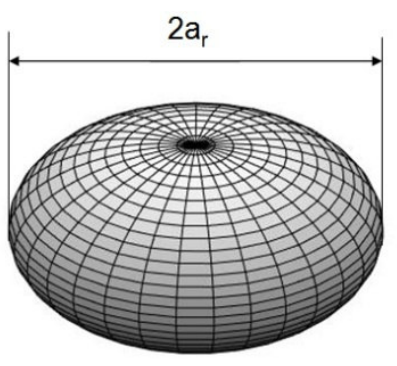

Oblate spheroid

Figure 1. Drop shapes and definition of semi-axes $a_{z}$ and $a_{r}$.

For spheroidal drops, an equivalent radius $R_{0}$ can be defined as the radius of a spherical drop having the same volume, and the constant $a$ in equations (8) can be related to this radius by:

$a=R_{0} \frac{\left|1-\varepsilon^{2}\right|^{1 / 2}}{\varepsilon^{1 / 3}}$

where the eccentricity parameter $\varepsilon$ is defined as the ratio between the axial and radial drop semi-axes (see again figure 1).

An analytical solution of equation (3) has the following form, for the prolate, oblate and spherical drops: 


$$
\begin{array}{ll}
G=b_{0} \ln \frac{\zeta-1}{\zeta+1}+\sum_{n=1}^{\infty} b_{n} P_{n}(\eta) Q_{n}(\zeta)+G_{\infty} & \text { (prolate) } \\
G=c_{0}\left(\arctan (\zeta)-\frac{\pi}{2}\right)+\sum_{n=1}^{\infty} c_{n} P_{n}(\eta) Q_{n}(i \zeta)+G_{\infty} & \text { (oblate) } \\
G=d_{0} \zeta+\sum_{n=1}^{\infty} d_{n} P_{n}(\eta) \zeta^{n+1}+G_{\infty} & \text { (sphere) }
\end{array}
$$

where $P_{n}$ and $Q_{n}$ are the Legendre functions of the first and second kind, respectively. The conditions at drop surface and at infinity:

$G\left(\zeta=\zeta_{0}, \eta\right)=G_{s}(\eta) ; \quad G(\zeta=\infty, \eta)=G_{\infty}$

allow to calculate the coefficients $b_{n}, c_{n}$ and $d_{n}$ and the constant $G_{\infty}$. The value of $G$ at infinity is constant since the vapour mass fraction is considered uniformly distributed at infinite distance from the drop, while on the surface the Dirichlet type conditions will be generally non-uniform and depending on the surface temperature conditions, since the saturation values of the mass fraction are correlated to the drop surface temperature and pressure and then:

$$
G_{s}(\eta)=\ln \left(1-\frac{P_{v, s a t} M m^{(1)}}{P_{v, \text { sat }}\left(M m^{(1)}-M m^{(0)}\right)+P_{T} M m^{(0)}}\right)
$$

The boundary conditions for the energy equation (5), again of Dirichlet type, are:

$T\left(\zeta=\zeta_{0}, \eta\right)=T_{s}(\eta) ; T(\zeta=\infty, \eta)=T_{\infty}$

The temperature distribution along the drop surface is chosen to be a polynomial of even degree (to satisfy the symmetry requirement across $\eta=0$ ). A fourth degree polynomial is the simplest choice to fit the values at $\eta=0$ and $\eta=1$ and to satisfy the further symmetry requirement: $\left.\frac{\partial T}{\partial \eta}\right)_{\eta=1}=0$.

The surface distribution of $G_{s}$ (equation 12) is approximated by a polynomial of even degree (again to satisfy symmetry condition across $\eta=0$ ) and the coefficients are calculated to satisfy the further symmetry condition on $\eta=1$ and to fit the values at $\eta=0$ and $\eta=1$. The choice of a polynomial form for $G_{s}$ allows to reduce the number of terms needed in equation (10). Since a polynomial of degree $p$ can be always written as a sum of the first $p$ Legendre polynomials, only the first $p$ coefficients in the series expansion (12) are different from zero.

\section{Solution of the energy equation}

Currently, to the best knowledge of the Authors, no analytical solution of the energy equation (5) satisfying general non-uniform boundary conditions at the drop surface is available (the simple solution $T=A e^{G(\zeta, \eta) / L e}+B$ of equation (5) cannot satisfy general non-uniform boundary conditions on the drop surface) and the energy problem is solved numerically, implementing a finite difference scheme on the rectangular computational grids $\eta \in(0,1) \times$ $\zeta \in\left(\zeta_{0}, \zeta_{\max }\right)$. Tests were performed to choose a proper value of $\zeta_{\max }$ to make the solution practically independent of it. Grid refinement was performed in the drop surface vicinity introducing a proper stretching function, which was optimised by comparison to the analytical solution of the energy equation available for uniform surface temperature condition (see [9]). Grid independence tests were performed and a final grid of 2000 cells proved to be enough to assure a sufficient grid independence of the solution and particularly of the fluxes on the drop surface.

\section{Results and discussions}

This section reports and comments the results obtained by implementing the model described in the previous paragraph, focusing on the effect of non-uniform temperature boundary conditions for the case of prolate drops. The choice of a prolate drop is related to the fact that for such shape it was shown [14] that the uneven heat flux caused by the variable curvature produces, during drop heating and evaporation, an uneven distribution of temperature over the surface. However, similar results can be obtained for the case of an oblate drop and even for the spherical drop.

The values of the fluid properties are evaluated at reference conditions (temperature and mass fraction) by the "1/3 rule" [15]: 
$T_{\text {ref }}=\frac{2 \bar{T}_{s}+T_{\infty}}{3} ; \chi_{\text {ref }}=\frac{2 \bar{\chi}_{s}+\chi_{\infty}}{3}$

Figure 2(a) shows qualitatively a sample of the vapour field around a prolate water drop with the eccentricity parameter $\varepsilon$ equal to 2.5; the surrounding gas temperature at free stream condition is fixed equal to $700 \mathrm{~K}$, the vapour mass fraction at infinity is taken equal to zero and the average surface temperature of the drop is equal to $340 \mathrm{~K}$, while seven different surface temperature profiles are imposed, defined by the maximum temperature difference at the drop surface as follows:

$\Delta T=T_{s}\left(\zeta_{0}, \eta=1\right)-T_{s}\left(\zeta_{0}, \eta=0\right)$

(a) Prolate drops, $\varepsilon=2.5$

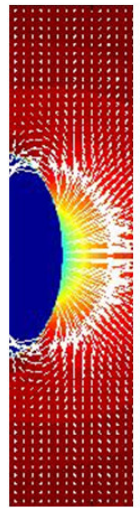

$\Delta T=-40 \mathrm{~K}$

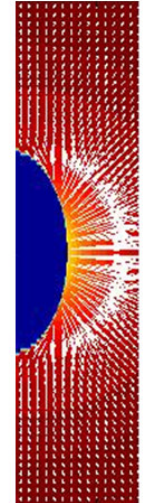

$\Delta T=-20 \mathrm{~K}$

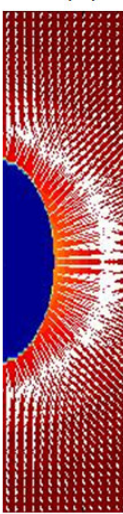

$\Delta T=-10 \mathrm{~K}$

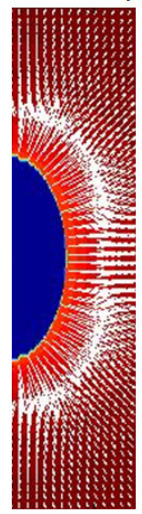

$\Delta T=0 \mathrm{~K}$

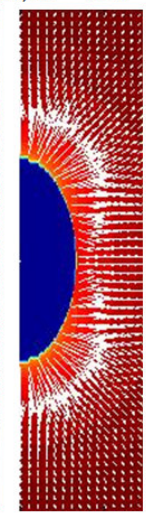

$\Delta T=10 \mathrm{~K}$

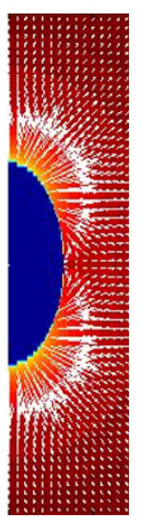

$\Delta T=20 \mathrm{~K}$

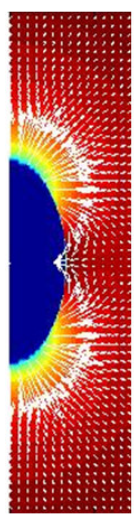

$\Delta T=40 \mathrm{~K}$

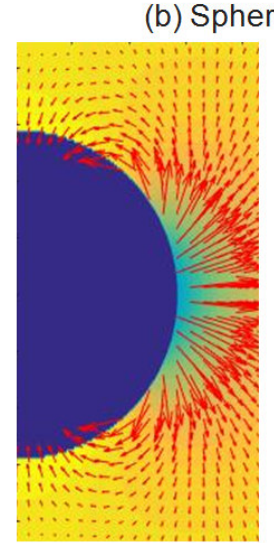

$\Delta T=-40 \mathrm{~K}$

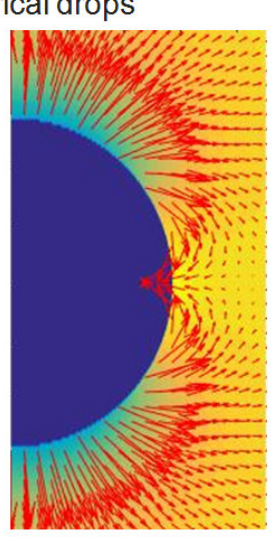

$\Delta T=40 \mathrm{~K}$

Figure 2. $G$-field and vapour flux around (a) prolate $(\varepsilon=2.5)$ and (b) spherical water drops, for different surface temperature profiles; $T_{\infty}=700 \mathrm{~K}, G_{\infty}=0, \bar{T}_{s}=340 \mathrm{~K}$.

The flow field is substantially affected by the non-uniform temperature boundary conditions at the drop surface, since vapour recirculation can be observed when the local surface temperature drops below the dew point value of the surrounding gas. The effect is mainly related to the temperature distribution and it is not peculiar of deformed drops, figure 2(b) shows the vapour distribution and flux around a spherical drop having a non-uniform surface temperature, and similar paths can be observed.

The calculated vapour distribution (equation 10a) is then used to numerically solve equation (5) and the local surface heat flux can be calculated. Figure $3(a)$ shows the heat fluxes, non-dimensionalised by the factor $\frac{2 R_{0}}{\left(\bar{T}_{s}-T_{\infty}\right) k}$ for the case of a prolate drop $(\varepsilon=2.5)$ while figure $3(\mathrm{~b})$ shows the local Nusselt number, defined as:

$$
N u(\eta)=\frac{\varphi(\eta) 2 R_{0}}{\left[T_{s}(\eta)-T_{\infty}\right] k}
$$

along the surface. The case $\Delta T=0$ is the one with uniform surface temperature and for this case the function $N u(\eta)$ can be found analytically (see [14]), showing that the surface curvature itself influences this parameter. The results for $\Delta T \neq 0$ show that the surface heat flux is strongly influenced by the non-uniform temperature distribution (see figure 3a). The local Nusselt number is also influenced by the non-uniform temperature distribution, but to a lesser extent, specially close to the "pole" $(\eta=1)$ where the effect of the different temperature distribution is lower (figure $3 b)$. 
(a)

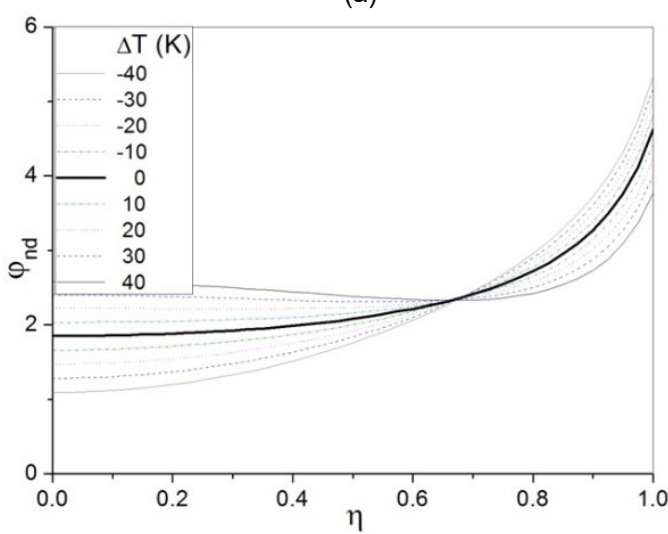

(b)

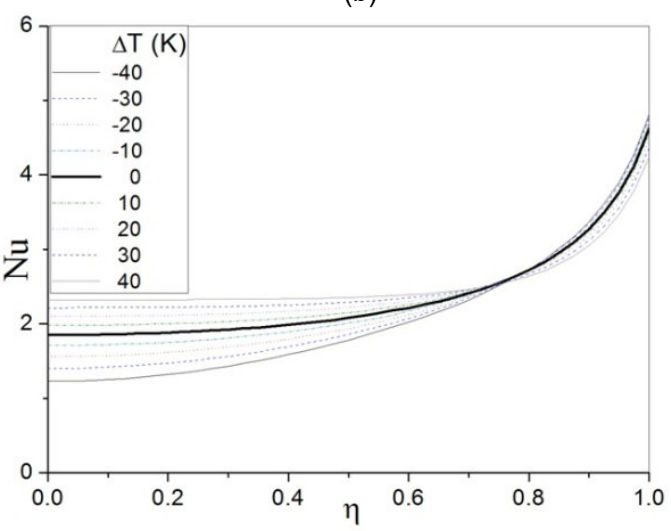

Figure 3. (a) Local vapour flux and (b) local Nusselt number along the drop surface for various surface temperature profiles (water drop, $\bar{T}_{s}=320 \mathrm{~K}, T_{\infty}=500 \mathrm{~K}$ ).

These effects are clearly not peculiar of one particular species but their magnitude depends on the thermophysical characteristics of the species. Calculations were performed selecting five different species, whose properties are reported in Table 1. These species represent some of the most common working fluid used for spray modelling in industrial applications.

\begin{tabular}{l|lll}
\hline \hline Species & $M m(\mathrm{~kg} / \mathrm{kmol})$ & $T_{b, n}(\mathrm{~K})$ & $L\left(T_{b, n}\right)(\mathrm{kJ} / \mathrm{kg})$ \\
\hline \hline water $\mathrm{H}_{2} \mathrm{O}$ & 18.02 & 373.15 & 2257.4 \\
ethanol $\mathrm{C}_{2} \mathrm{H}_{6} \mathrm{O}$ & 46.07 & 351.39 & 850.53 \\
acetone $\mathrm{C}_{3} \mathrm{H}_{6} \mathrm{O}$ & 58.08 & 329.22 & 501.85 \\
n-octane $\mathrm{C}_{8} \mathrm{H}_{18}$ & 114.23 & 398.80 & 301.10 \\
n-dodecane $\mathrm{C}_{12} \mathrm{H}_{26}$ & 179.34 & 489.50 & 256.70
\end{tabular}

Table 1. Molar mass, normal boiling temperature and latent heat of evaporation for the selected liquid species.

The profiles of the local Nusselt number $(\mathrm{Nu})$ and the percentage deviation $\Delta \%$ from the values obtained with a surface temperature uniformly equal to the mean temperature are presented in Fig. 4 for each fluid.

These results were obtained maintaining constant the drop deformation $(\varepsilon=2.5)$ and the gas temperature $\left(T_{\infty}=500 \mathrm{~K}\right)$ and setting the drop mean surface temperature equal to the corresponding quasi-steady (sometimes called plateau or asymptotic [16]) temperature. The deviations from the uniform temperature case are relatively small for water and n-dodecane while they are more relevant for acetone and n-octane. The different behaviour is due to a combination of the effects of boiling temperature and latent heat of vaporisation. These peculiarities should be considered when approximating the heat transfer coefficient from the analytic relation that can be deduced from the uniform surface temperature analysis $[9,14]$.

The drop deformation has an effect on the deviation of the local Nusselt number from the values for the uniform temperature case, as reported in figure 5. The increase of the deformation decreases the deviation, which should be expected since the effect of curvature becomes dominant over the effect of temperature non-uniformity when $\varepsilon$ increases.

Also the increase of the gas temperature has a similar effect (see figure 6), since in this case the relative nonuniformity of the temperature difference between drop and gas decreases, leaving again the curvature as the dominant parameter.

Finally the evaporation rates and the sensible heat rate, calculated integrating the species and heat fluxes over the drop surfaces, are reported in figure 7 as a function of the temperature non-uniformity for the case of $n$ dodecane. The temperature non-uniformity has a non neglectful effect on the evaporation rate, which mainly increases when the temperature becomes non-uniform, reaching relative variation of about $30 \%$ for the case $\Delta T=-40^{\circ} \mathrm{C}$, with a peculiar behaviour for small temperature non-uniformity, which may cause a small decrease of the evaporation rate (around $1 \%$ ) when $\Delta T \approx+10^{\circ} \mathrm{C}$. The heat rate instead increases for positive values of $\Delta T$ and decreases for negative values, but the maximum differences are lower than $8 \%$. 

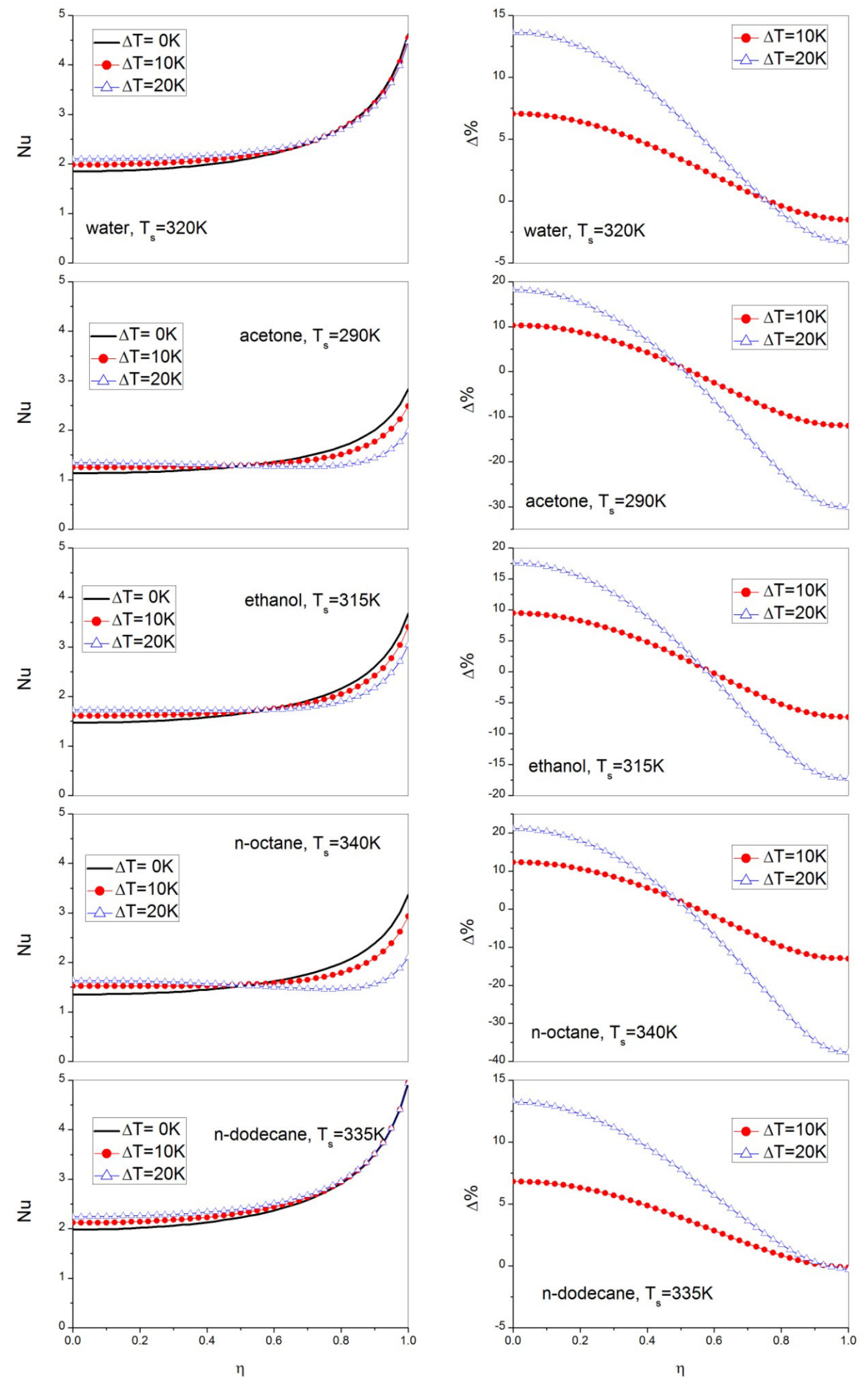

Figure 4. Local Nusselt number $(\mathrm{Nu})$ and percentage deviation $(\Delta \%)$ from the uniform surface temperature case for five species: water, acetone, ethanol, $\mathrm{n}$-octane, $\mathrm{n}$-dodecane, for different $\Delta T\left(\varepsilon=2.5, T_{\infty}=500 \mathrm{~K}, T_{s}=T_{\text {plateuu }}\right)$.

This work is licensed under a Creative Commons 4.0 International License (CC BY-NC-ND 4.0). 
(a)

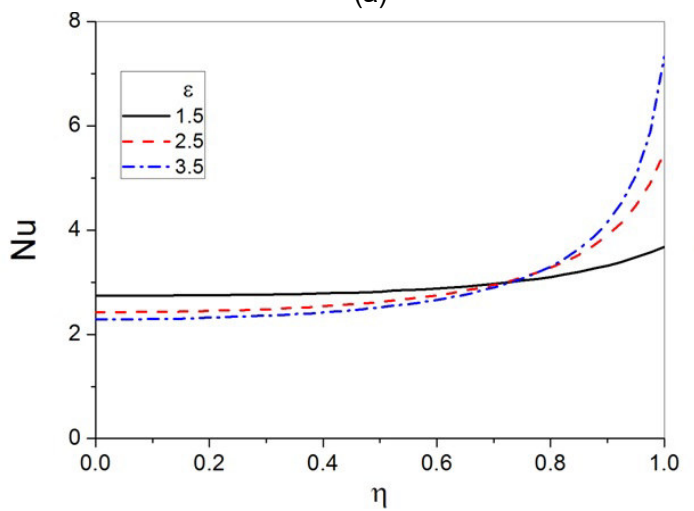

(b)

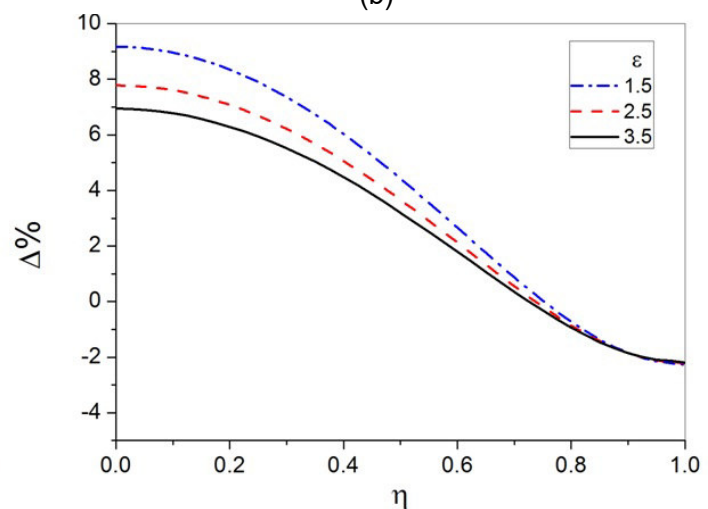

Figure 5. Local Nusselt number $(N u)$ and percentage deviation ( $\triangle \%)$ (figures (a) and (b) respectively) for different values of $\varepsilon(\mathrm{n}$ dodecane, $\left.T_{\infty}=700 \mathrm{~K}, T_{s}=380 \mathrm{~K}, \Delta T=20 \mathrm{~K}, P=10^{5} \mathrm{~Pa}\right)$.

(a)

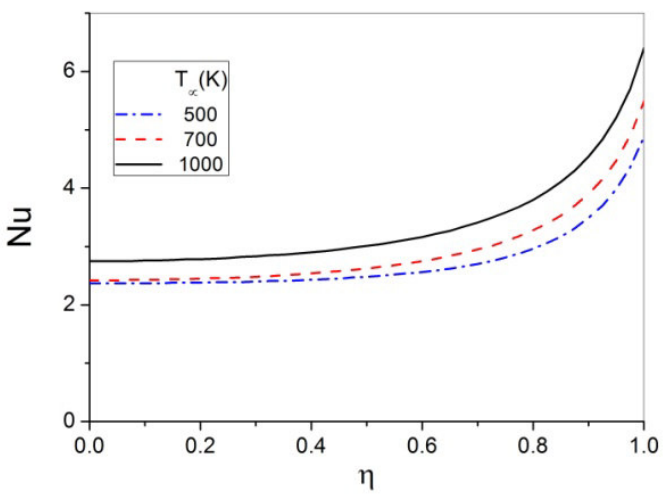

(b)

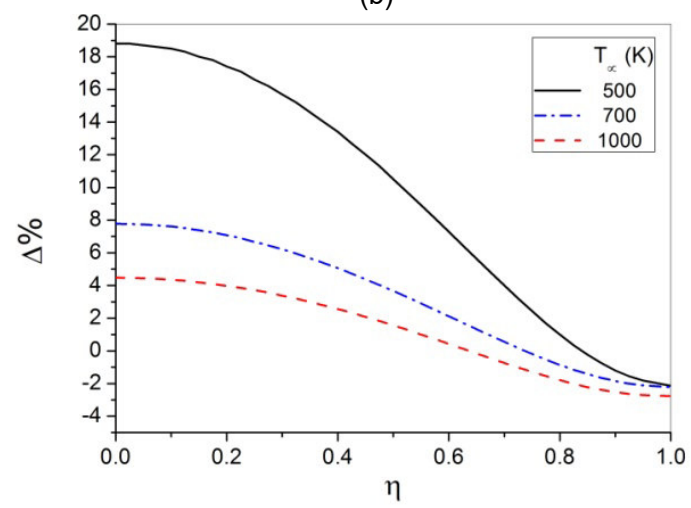

Figure 6. Local Nusselt number $(N u)$ and percentage deviation ( $\Delta \%$ ) (figures (a) and (b) respectively) for different $T_{\infty}(\mathrm{n}$ dodecane, $\left.\varepsilon=2.5, T_{s}=380 \mathrm{~K}, \Delta T=20 \mathrm{~K}, P=10^{5} \mathrm{~Pa}\right)$.

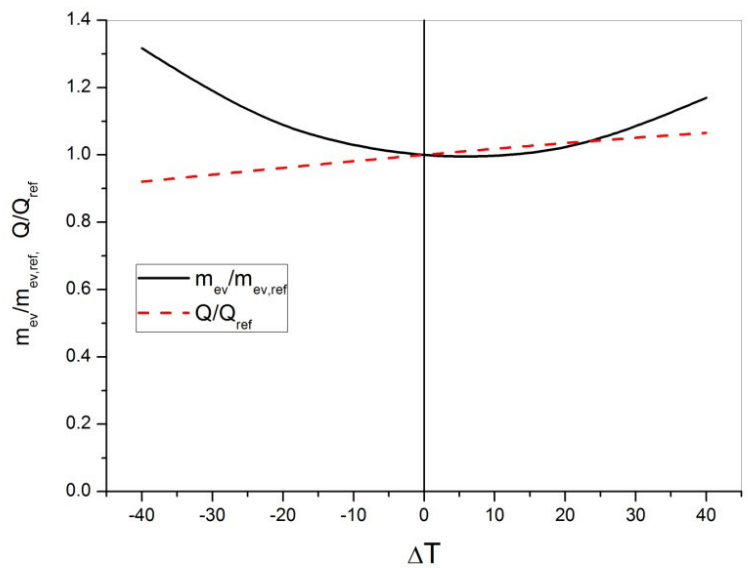

Figure 7. Evaporation rate and heat rate for a n-dodecane prolate drop $(\varepsilon=2.5)$ as a function of the temperature profile; the values are non-dimensionalised by the respective values for the uniform surface temperature case.

\section{Conclusions}

The effect of temperature non-uniformity on the heating and evaporation of spherical and spheroidal drops in a still gaseous environment are investigated analytically and numerically. The species conservation equations are analytically solved in proper coordinate systems (spherical or spheroidal) and the energy equation is numerically solved in the same coordinate system. 
A detailed analysis is reported for the case of a prolate evaporating drop, accounting for different species (water, acetone, n-octane, ethanol, n-dodecane), different gas temperature, drop deformation and temperature nonuniformity.

The effects on the local mass flux can become quite important, with local re-circulations when the temperature differences between the drop pole and equator become relative large $\left(20\right.$ to $\left.40^{\circ} \mathrm{C}\right)$.

The local Nusselt number is found to depend on local curvature and temperature, although for not too large values of the maximum surface temperature variation (less than $20^{\circ} \mathrm{C}$ ) the effect of curvature prevails and the analytical values obtained for the uniform temperature case are still usable with acceptable errors.

The local heat transfer coefficient is less affected by the non-uniformity of surface temperature when the deformation is large and when the gas temperature increases.

The total evaporation rate is affected by the temperature distribution and can increase up to $30 \%$ when drop nonuniformity is increased up to $40^{\circ} \mathrm{C}$, still maintaining the same average surface temperature. The heat rate is less affected showing deviation less than $8 \%$ for temperature difference between drop pole and equator as large as $40^{\circ} \mathrm{C}$.

\section{References}

[1] Mashayek, F., 2001, International Journal of Heat and Mass Transfer, 44(8), pp. 1517-1526.

[2] Haywood, R. J., Renksizbulut, M., and Raithby, G. D., 1994, International Journal of Heat and Mass Transfer, 37(9), pp. 1401-1409.

[3] Loth, E., 2008, International Journal of Multiphase Flow, 34(6), pp. 523-546.

[4] Hase, M., Weigand, B., 2004, Transient heat transfer of deforming droplets at high Reynolds numbers, International Journal of Numerical Methods for Heat and Fluid Flow, 14(1), pp. 85-97.

[5] Schlottke, J., Weigand, B., 2008, Direct numerical simulation of evaporating droplets, Journal of Computational Physics, 227, pp. 5215--5237.

[6] Mashayek, F., 2001, International Journal of Heat and Mass Transfer, 44, pp. 1527-1541.

[7] Tonini, S., Cossali, G.E., 2013, International Journal of Heat and Mass Transfer, 60, pp. 236-240.

[8] Tonini, S., Cossali, G.E., 2014, International Journal of Heat and Mass Transfer, 51, pp. 18-24.

[9] Tonini, S., Cossali, G.E., 2016, International Journal of Heat and Mass Transfer, 97, pp. 301-307.

[10] Abramzon, B., Sirignano, W.A., 1989, International Journal of Heat and Mass Transfer, 32 (9), pp. 16051618.

[11] Abramzon, B., Sazhin, S., 2006, Fuel, 85 (1), pp. 32-46.

[12] Sazhin, S., Krystadi, T., Abdelghaffar, W.A., Heikal, M.R., 2006, Fuel, 85 (12-13), pp. 1613-1630.

[13] Al Quebeissi, M., PhD Thesis, University of Brighton (2015).

[14] Zubkov, V.S., Cossali, G.E., Tonini, S., Rybdylova, O., Crua, C., Heikal, M., Sazhin, S.S., 2017, International Journal of Heat and Mass Transfer, 108, pp. 2181-2190.

[15] Yuen, M.C., Chen, L.W., 1976, Combustion Science and Technology, 14, pp. 147-154.

[16] Tonini, S., Cossali, G.E., 2014, International Journal of Thermal Sciences, 75, pp. 194-203.

\section{Nomenclature}

Roman symbols

$a \quad$ length scale in spheroidal coordinate [m]

$b_{n}, c_{n}, d_{n} \quad$ constants, eqs. (10) [-]

$c_{p, v} \quad$ vapour heat capacity $[\mathrm{J} / \mathrm{kgK}]$

$D_{10} \quad$ mass diffusivity $\left[\mathrm{m}^{2} / \mathrm{s}\right]$

$G \quad$ non-dimensional function [-]

$k \quad$ thermal conductivity [W/mK]

Le Lewis number [-]

$m_{e v} \quad$ evaporation rate $[\mathrm{kg} / \mathrm{s}]$

$M_{m} \quad$ molar mass $[\mathrm{kg} / \mathrm{kmol}]$

$n_{v} \quad$ mass flux $\left[\mathrm{kg} / \mathrm{sm}^{2}\right]$

Greek symbols

$\varepsilon \quad$ drop eccentricity parameter [-]

$\chi \quad$ mass fraction [-]

$\gamma, \eta, \zeta \quad$ spheroidal coordinate system [-]

Subscripts

$k, p \quad$ index [-]

ref reference case [-]
$\mathrm{Nu} \quad$ Nusselt number [-]

$P_{n} \quad$ Legendre function of first kind [-]

$P_{v, s a t} \quad$ saturation vapour pressure [Pa]

$P_{T} \quad$ total pressure [Pa]

$Q_{n} \quad$ Legendre function of second kind [-]

$Q \quad$ heat rate [W]

$R_{0} \quad$ drop radius [m]

$T \quad$ temperature $[\mathrm{K}]$

$U \quad$ Stefan velocity $[\mathrm{m} / \mathrm{s}]$

$x, y, z \quad$ coordinate system [-]

$\varphi \quad$ heat flux $\left[\mathrm{W} / \mathrm{m}^{2}\right]$

$\rho \quad$ density $\left[\mathrm{m}^{3} / \mathrm{kg}\right]$

$v \quad$ vapour [-]

$\infty \quad$ at infinite [-] 